\title{
Polyetherimide Nanofibres as Sorbents for Organochlorinated Pesticides Determination
}

\author{
Antoš Vojtěch, Hrabák Pavel, Komárek Michal, and Stuchlík Martin \\ Technical University of Liberec, CxI, Studentská 2, 46117 Liberec, Czech Republic \\ Correspondence should be addressed to Antoš Vojtěch; vojtech.antos@tul.cz
}

Received 24 March 2016; Revised 27 April 2016; Accepted 1 June 2016

Academic Editor: Marco Salerno

Copyright (C) 2016 Antoš Vojtěch et al. This is an open access article distributed under the Creative Commons Attribution License, which permits unrestricted use, distribution, and reproduction in any medium, provided the original work is properly cited.

Electrospun polyetherimide (PEI) nanofibres were fixed on a steel wire solid phase microextraction (SPME) assembly. The basic properties of the prepared nanofibres were determined by thermogravimetry, differential scanning calorimetry, adsorption, and SEM. The analytical performance of prepared PEI SPME fibres was compared with three commercially available SPME fibres, $7 \mu \mathrm{m}$ PDMS, $100 \mu \mathrm{m}$ PDMS, and DVB/Carboxene/PDMS. As model water pollutants, persistent organochlorinated pesticides hexachlorocyclohexanes $(\mathrm{HCH})$ and chlorobenzene $(\mathrm{ClB})$ were chosen as model water pollutants. The fibres were compared in the headspace- (HS-) SPME mode of GC-MS/MS instrumentation. The comparison omitted other method parameters and focused exclusively on the extraction time variability. Lab-made PEI SPME fibres showed significantly better response for the target compounds than the other tested fibres from industrial production. Based on the results, the extraction time could be shortened from 50 to $10 \mathrm{~min}$, if PEI SPME fibres were used as a modification of existing analytical protocol.

\section{Introduction}

Headspace solid phase microextraction (HS-SPME) is a modern analytical technique for sample introduction into gas chromatographic systems [1]. The HS-SPME technique combines extractive sorption (enrichment) of targets to be quantified with ongoing thermal desorption and injection to the GC system. All of the above-mentioned steps can be performed by an ordinary autosampler [2]. This fact makes the technique attractive, as it meets the current laboratory demands on high sample throughput, precision, and green analytical approach as solventless operation [3]. With the progress in automation of sample preparation steps, HS-SPME is often involved in wider protocols of coupled techniques such as on-fibre derivatization $[4,5]$.

SPME fibres are commercially available in various geometries: as polymeric coatings on a stainless steel or on a glass core, as polymeric cylinders with a surface membrane, as needle trap devices, and so forth $[6,7]$. The most often routinely employed polymers are polydimethylsiloxane (PDMS), divinylbenzene (DVB), polyacrylate (PA), or polyethylene glycol (PEG) [8]. Polypyrrole [9], cyclodextrin [10], polymeric ionic liquids [11], or graphene-based [12] materials were proposed to become the next generation of SPME sorbents.

A syringe-like SPME fibre geometry appears to be most viable: polymeric coatings, sandwiches, and mixtures are placed at the tip of the plunger, which is either hidden in a needle (during the transport phase) or exposed off the needle (during the extraction and thermal desorption phases of the analysis). The usual length of the plunger tip is $10 \mathrm{~mm}$ and its diameter is $0.1 \mathrm{~mm}$. Polymeric layer thickness ranges from 7 to $100 \mu \mathrm{m}$. It should be noted at this point that there is a vast amount of literature and running research on SPME fibres prepared by sol-gel based techniques (see, e.g., the review by Kumar et al. [13]), which are commercially available, for example, from Supelco or CTC Analytics, AG. Our study contributes to the fresh technique of making SPME assemblies from nanofibres. First analytically successful preparations of electrospun SPME assemblies were announced few years earlier: in 2012 for polyamide [14]; in 2014 for polyacrylate-polyethylene glycol copolymer (PAPEG) [15] and for polyetherimide (PEI) [16]. The latter polymer is also the topic of this research. 
Amongst other parameters, the duration of the extractive sorption part of the sample introduction step (enrichment) seems to highly influence the overall time of analysis. This is especially the case if less volatile organic compounds are the target analytes. Various solutions are proposed to avoid the long enrichment times: First of all, the salting out effect is utilized for water samples whereby $\mathrm{NaCl}$ addition of up to $10 \%$ solution is used [17]. Temperature is another parameter driving the analyte transfer velocity from the water phase towards the sorption fibre. However, water vapour competes with organic analytes vapours for sorption places at higher temperatures [18]; therefore, temperatures exceeding $80^{\circ} \mathrm{C}$ are rarely used even for organic compounds with a low Henry constant. Last but not least, sorption fibre polymeric material affinity for specific analytes and its surface preconditioning play an important role [19].

Polyetherimide (PEI) nanofibres were chosen as the polymeric surface of the lab-made steel core SPME assemblies to be evaluated in our study. During the production of PEI nanofibres by needle electrospinning, a lab-made SPME plunger was rotated inside a stream of freshly spewed PEI nanofibres in order to obtain a surface covering. Completed SPME assemblies were compared with commercial PDMS and DVB/Carboxene/PDMS fibres with an emphasis on the possible shortening of the duration of the enrichment step. As a matrix for enrichment time tests, water contaminated by hexachlorocyclohexanes $(\mathrm{HCH})$ and chlorobenzene $(\mathrm{ClB})$ was used. These compounds were selected: (a) as they belong to the group of persistent organochlorinated pesticides listed as POPs under the Stockholm Convention on persistent organic pollutants and have therefore priority attention, (b) as there is a need to perform detailed monitoring of the environmental contamination of $\mathrm{HCH}$ and $\mathrm{ClB}$ due to their former industrial production in the Czech Republic [20], and (c) as organochlorinated pesticides belong to widely studied groups of target analytes with SPME utilization [21-25]. $\mathrm{HCH}$ are always accompanied by $\mathrm{ClB}$ in groundwater, as $\mathrm{ClB}$ are products of $\mathrm{HCH}$ natural transformations [20]. Aside from characterization of PEI SPME analytical performance by GC-MS/MS system response, the prepared assemblies were characterized by thermogravimetry (TGA), differential scanning calorimetry, adsorption (BET isotherm), and scanning electron microscopy (SEM).

\section{Materials and Methods}

2.1. Materials and Reagents. Lab-made SPME fibres were assembled from a stainless steel capillary and $304 \mathrm{H}$ wire supplied by Teseco and RDG810 3D-printer polymer supplied by VeroClear (Figure 2). PEI was obtained from Sigma-Aldrich (CAS: 61128-46-9, melt index $18 \mathrm{~g} / 10 \mathrm{~min}$, $337^{\circ} \mathrm{C} / 6.6 \mathrm{~kg}$ ) and was dissolved overnight in a mixture of dimethylformamide/tetrahydrofuran (Sigma-Aldrich) $80: 20$ into $15 \% \mathrm{w} / \mathrm{w}$ solution (labelled S1) for electrospinning, following the procedure of Bagheri et al. [16].

Chromatographic standards of hexachlorocyclohexanes $(\mathrm{HCH})$, Mix $5\left(100 \mu \mathrm{g} \cdot \mathrm{mL}^{-1}\right.$ in acetone), and chlorobenzene (ClB), Mix $12\left(1000 \mu \mathrm{g} \cdot \mathrm{mL}^{-1}\right.$ in acetone), were purchased from Neochema; isotopically labelled $\gamma$-HCH D6 $\left(100 \mu \mathrm{g} \cdot \mathrm{mL}^{-1}\right.$ in cyclohexane) was obtained from Dr. Ehrenstorfer $\mathrm{GmbH}$ (Table 1). SPME commercial fibres were obtained from Supelco (100 $\mu \mathrm{m}$ PDMS and 50/30 $\mu \mathrm{m}$ DVB/ Carboxene/PDMS) and CTC Analytics AG (7 $\mu \mathrm{m}$ PDMS).

Helium of 5.0 purity and argon of 4.8 purity (Linde Gas) were used as a carrier gas and collision gas for GC-MS/MS measurements, respectively. Argon and nitrogen gases of 4.8 purity (Linde Gas) were used for surface area determination. Low purity nitrogen was used for the thermogravimetric and calorimetric measurements.

2.2. Apparatus and Equipment. Mass loss temperature dependence of $100 \mu \mathrm{m}$ PDMS and PEI fibres was compared by measurements using TGA Q500 (Thermo). Fibres were tested under nitrogen flow (60 $\mathrm{mL}$ per $\mathrm{min})$, which resembles GC injector inert conditions rather than air composition. The temperature program was started at $25^{\circ} \mathrm{C}$, graduating by $10^{\circ} \mathrm{C}$ per minute to $750^{\circ} \mathrm{C}$, with a hold for $60 \mathrm{~min}$ at $250^{\circ} \mathrm{C}$.

The appearance of manufactured PEI and Supelco PDMS fibres was documented using SEM microscope Tescan Vega 3 (Figure 2). The specific surface of electrospun PEI fibres was determined by the absorption method nitrogen/krypton upon liquid nitrogen temperature and was evaluated with a BET adsorption isotherm. Measurement was performed on an Autosorb IQ-MP instrument (Quantachrome Instruments).

The temperature of glass transition was determined by differential scanning calorimetry (Mettler Toledo DSC1). The temperature was program started at $0^{\circ} \mathrm{C}$, graduating by $10^{\circ} \mathrm{C}$ per minute to the final temperature of $250^{\circ} \mathrm{C}$. The flow of carrier gas (nitrogen) was adjusted to $50 \mathrm{~mL}$ per minute. This program was repeated twice, firstly to remove sample thermal history and secondly to determine the real physical properties of the PEI nanofibres.

Analytical performance of all SPME fibres was tested on a gas chromatograph (Thermo Trace 1310) equipped with a mass spectrometer triple quadrupole detector (Thermo $\mathrm{TSQ}^{\mathrm{TM}} 8000$ ), autosampler (CTC Analytics AG, PAL LHX-xt), and a programmed temperature vapourizing injector (PTV). For the $4.5 \mathrm{~min}$ long fibre conditioning step, the PTV was set at $255^{\circ} \mathrm{C}$ in the case of the commercial fibres and at $205^{\circ} \mathrm{C}$ for lab-made PEI fibres. The split flow was set at $50 \mathrm{~mL}$ per min for the conditioning phase. For the $1 \mathrm{~min}$ long desorption and injection step, the PTV was set at $200^{\circ} \mathrm{C}$ in splitless operation.

A VF-5MS chromatographic column (30 m long, $0.25 \mathrm{~mm}$ thick with a stationary phase thickness of $0.25 \mu \mathrm{m}$, Agilent) was installed in the gas chromatograph. The temperature program of the chromatographic oven started at $60^{\circ} \mathrm{C}$, graduating firstly by $10^{\circ} \mathrm{C}$ per min to $250^{\circ} \mathrm{C}$, followed by a temperature gradient of $35^{\circ} \mathrm{C}$ per min to $300^{\circ} \mathrm{C}$ and held on this temperature for $3 \mathrm{~min}$. The carrier gas flow was adjusted to $1 \mathrm{~mL}$ per min. All of the SPME fibres were tested for graduating extraction (enrichment) time in the headspace of the measured vials: for $1,2,3,4,5,10,30$, and 50 minutes at the same incubation temperature of $70^{\circ} \mathrm{C}$ and an agitator speed of $250 \mathrm{rpm}$. For each tested fibre, twenty-four tap water samples spiked with the same concentration of $\mathrm{HCH}$ and $\mathrm{ClB}$ standard $\left(2.5 \mu \mathrm{g} \cdot \mathrm{L}^{-1}\right.$ of each $\mathrm{HCH}$ isomer, $0.3 \mu \mathrm{g} \cdot \mathrm{L}^{-1}$ 
TABLE 1: Retention times and SRM transitions of the studied $\mathrm{HCH}$ and $\mathrm{ClB}$.

\begin{tabular}{|c|c|c|c|c|}
\hline Compound & $\begin{array}{l}\text { GC retention time } \\
\min \end{array}$ & $\begin{array}{c}\text { Precursor ion } \\
m / z\end{array}$ & $\begin{array}{c}\text { Product ion } \\
m / z\end{array}$ & $\begin{array}{c}\text { Collision energy } \\
\mathrm{eV}\end{array}$ \\
\hline 1,3-Dichlorobenzene & 5.88 & 146 & 75 & 20 \\
\hline 1,4-Dichlorobenzene & 6.01 & 146 & 75 & 20 \\
\hline 1,2-Dichlorobenzene & 6.30 & 146 & 75 & 20 \\
\hline 1,3,5-Trichlorobenzene & 7.75 & 180 & 109 & 20 \\
\hline 1,2,4-Trichlorobenzene & 8.43 & 180 & 109 & 20 \\
\hline 1,2,3-Trichlorobenzene & 8.93 & 180 & 109 & 20 \\
\hline $1,2,4,5+1,2,3,5$-Trichlorobenzene & 10.54 & 214 & 108 & 30 \\
\hline 1,2,3,4-Trichlorobenzene & 11.24 & 214 & 108 & 30 \\
\hline Pentachlorobenzene & 13.04 & 250 & 215 & 18 \\
\hline$\alpha-\mathrm{HCH}$ & 15.20 & 181 & 145 & 22 \\
\hline Hexachlorobenzene & 15.29 & 284 & 214 & 30 \\
\hline$\beta-\mathrm{HCH}$ & 15.75 & 181 & 145 & 15 \\
\hline$\gamma-\mathrm{HCH}$ & 15.93 & 181 & 145 & 22 \\
\hline$\gamma$-HCH D6 & 15.93 & 224 & 150 & 20 \\
\hline$\delta-\mathrm{HCH}$ & 16.52 & 181 & 145 & 24 \\
\hline$\varepsilon-\mathrm{HCH}$ & 16.73 & 217 & 181 & 8 \\
\hline
\end{tabular}

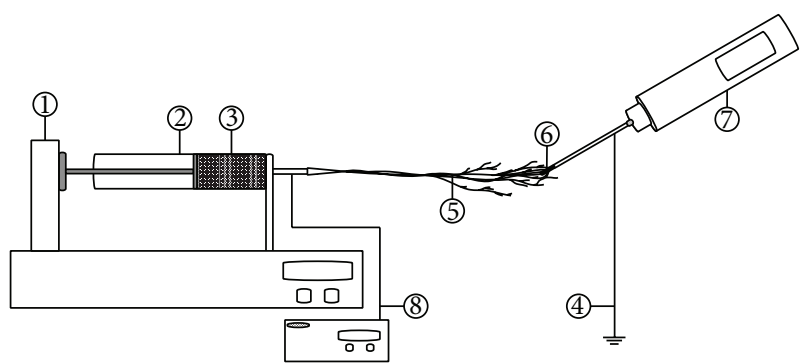
(1) Syringe pump
(2) Syringe
(3) Polymeric solution
(4) Grounded collector
(5) Nanofibres
(6) SPME fibre
(7) Motor
(8) High voltage power supply

FIGURE 1: The scheme of the needle electrospinning apparatus.

of deuterated $\gamma-\mathrm{HCH}$, and $0.6 \mu \mathrm{g} \cdot \mathrm{L}^{-1}$ of each $\mathrm{ClB}$ ) were prepared. Lower concentrations of $\gamma$-HCH D6 were used as they are established on this level as a GC-MS/MS internal standard for routine $\mathrm{HCH}$ determination via HS-SPME in our laboratory.

The retention times of all of the $\mathrm{HCH}$ and $\mathrm{ClB}$ isomers were determined previously in the full scan mode of MS detector and, subsequently, selected reaction monitoring (SRM) transitions were specified in order to enhance detector selectivity and sensitivity for $\mathrm{HCH}$ and $\mathrm{ClB}$ (Table 1).

\subsection{Methods}

2.3.1. Preparation of PEI Nanofibres. Solution $S 1$ was pumped into the electrospinner needle with a flow of $5 \mu \mathrm{L}$ per min, whereby the needle was connected at $16 \mathrm{kV}$. The grounded SPME plunger collected the freshly produced nanofibres at a speed of $50 \mathrm{rpm}$ at $25^{\circ} \mathrm{C}$ and relative humidity of $38 \%$ (Figure 1).

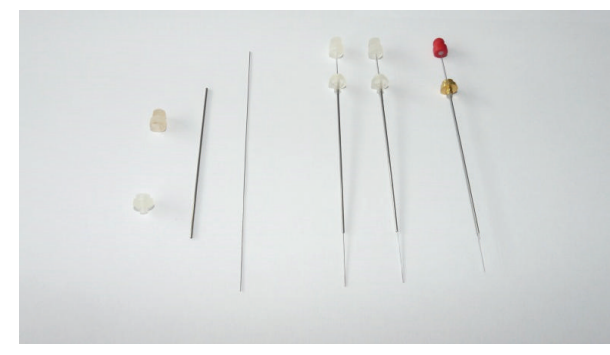

FIGURE 2: From left: plastic components, steel capillary and wire for lab-made fibre, 2 pieces of assembled lab-made fibres, and a commercial SPME fibre.

All of the commercial SPME fibres were conditioned according to the manufacturer's instruction before the measurements. Lab-made PEI fibres were conditioned at $200^{\circ} \mathrm{C}$ for 90 minutes in PTV injector with the flow of carrier gas set to $20 \mathrm{~mL}$ per min.

The analytical performance of the HS-SPME fibre was tested in $20 \mathrm{~mL}$ vials capped with PTFE/silicon septa and a magnetic cap, with $10 \mathrm{~mL}$ of the water sample. These method parameters together with chromatographic and MS settings were used as they had been previously validated on a GCMS/MS instrument for $\mathrm{HCH}$ and $\mathrm{ClB}$ determination in water with the legally required sensitivity (LOQ $0.01 \mu \mathrm{g} \cdot \mathrm{mL}^{-1}$ of $\gamma$ $\mathrm{HCH}$ and $0.02 \mu \mathrm{g} \cdot \mathrm{mL}^{-1}$ of the sum of $\mathrm{HCH}$ isomers).

\section{Results and Discussion}

For the purpose of this study, several pieces of SPME assemblies with plunger tips covered by electrospun PEI were prepared (Figures 2 and 3). Thermogravimetric characterization of both PDMS and PEI SPME fibres shows an insignificant difference in the temperature dependent mass 


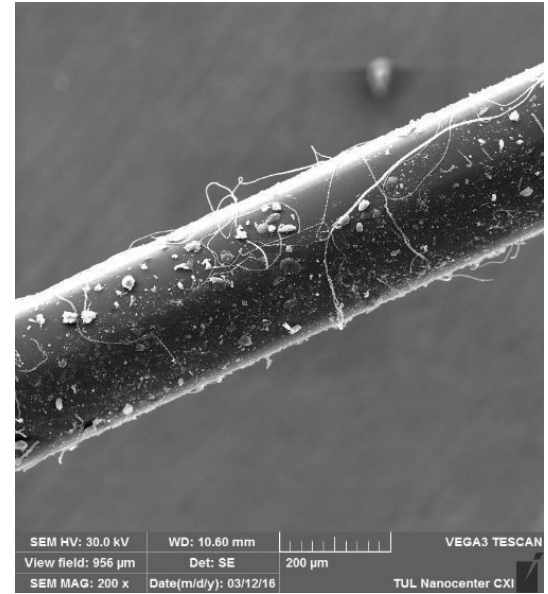

(a)

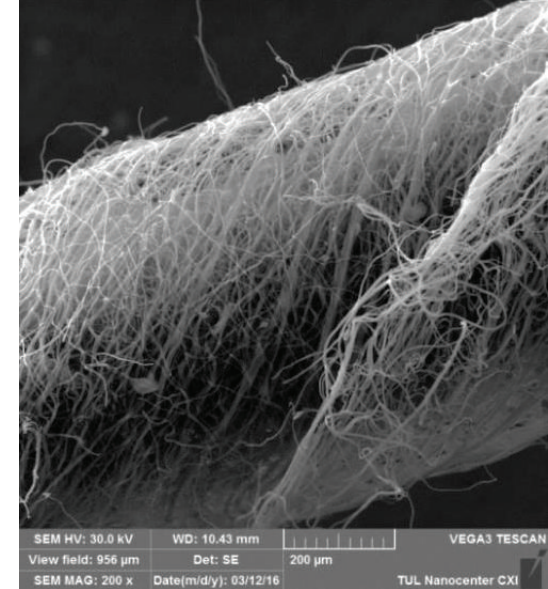

(b)

FIgURE 3: A spent $100 \mu \mathrm{m}$ PDMS fibre from Supelco (a) and a lab-made PEI fibre (b).
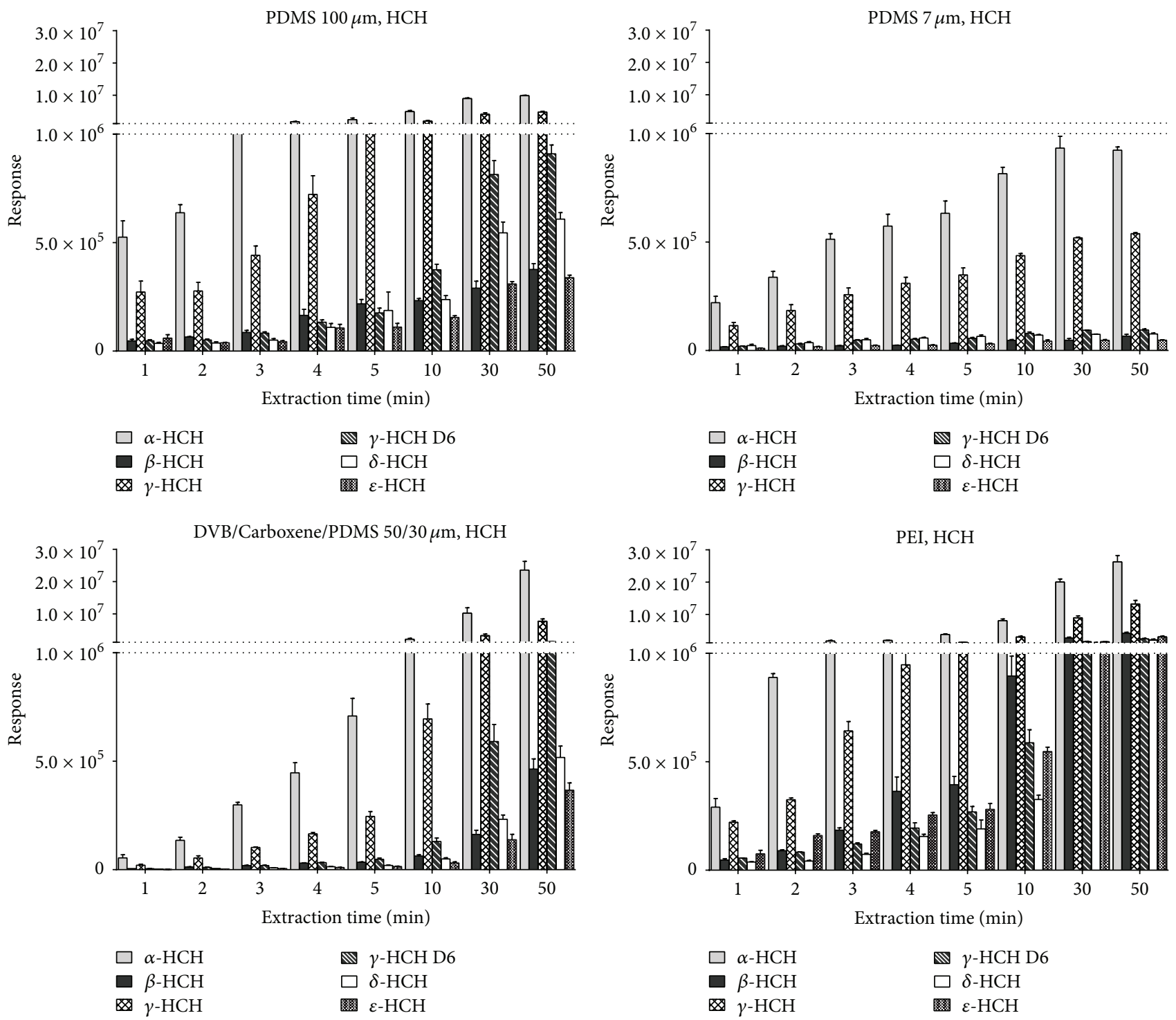

FIGURE 4: HCH response for 3 commercial and PEI fibres (the scale is interrupted at the response value of $1.0 \times 10^{6}$ on all of the graphs, error bars at $1 \sigma, n=3$ ). 

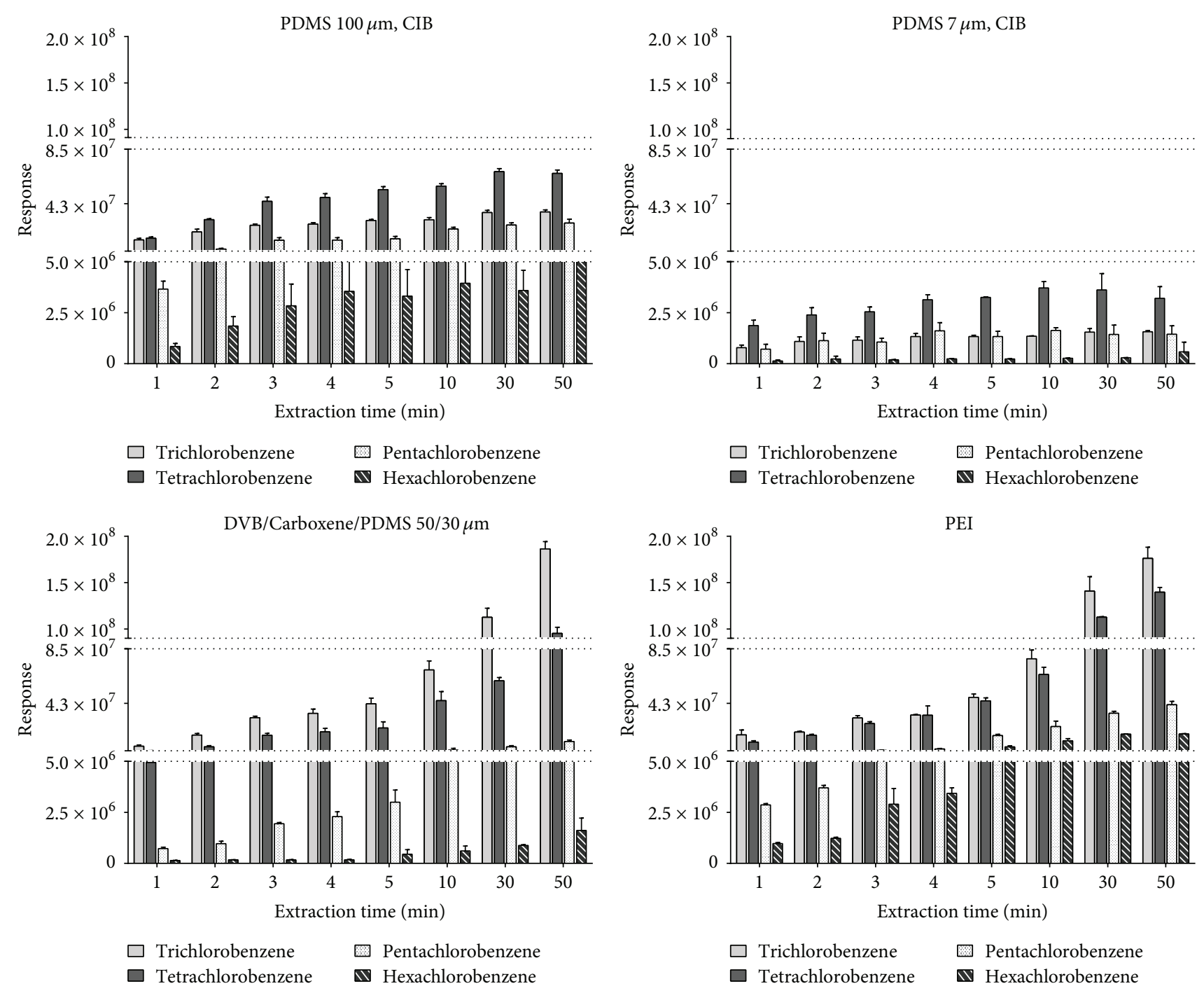

FIGURE 5: ClB response for 3 commercial and PEI fibres (the scale is interrupted at the response values of $5.0 \times 10^{6}$ and $8.5 \times 10^{7}$ on all of the graphs, error bars at $1 \sigma, n=3$ ).

loss. This was a prerequisite for fibre analytical utilization because polymer degradation products are not welcome in a GC-MS/MS system.

Calorimetric testing determined the glass transition temperature of PEI to approximately $210^{\circ} \mathrm{C}$. This explains the decrease in the sorption capacity of the PEI fibres, when an injector temperature of $250^{\circ} \mathrm{C}$ was used (data not shown). The data presented (Figures 4 and 5) were collected at an injector temperature of $200^{\circ} \mathrm{C}$.

The surface area was the last measured nonanalytical parameter of the prepared nanofibres. A high value of $58.1 \mathrm{~m}^{2}$. $\mathrm{g}^{-1}$ seemed promising in the effort to make the extractive (enrichment) phase the shortest one as possible.

The dependencies of GC-MS/MS peak areas on the extraction time for $6 \mathrm{HCH}$ isomers (including one isotopic labelled isomer) and for four groups of $\mathrm{ClB}$ are shown in Figures 4 and 5, respectively. For clarity, $\mathrm{ClB}$ data were grouped according to the degree of chlorination (sum of trichlorinated benzene, sum of tetrachlorinated benzene, pentachlorobenzene, and hexachlorobenzene). The DVB/Carboxene/PDMS fibre appeared to have the absolutely highest response values from commercial fibres. This fibre also did not approach the sorption equilibrium during $50 \mathrm{~min}$ of extraction time. Conversely, pure PDMS fibres either reached the sorption equilibrium after $50 \mathrm{~min}$ ( $7 \mu \mathrm{m}$ fibre) or were close to it $(100 \mu \mathrm{m}$ fibre). The responses at distinct extraction times were noticeably lower for PDMS fibres. In the case of $7 \mu \mathrm{m}$ PDMS fibre the response was only a few \% compared to all other fibres.

The PEI fibre performed similarly to the DVB/Carboxene/PDMS fibre in the meaning of high response. However, there are some differences between the PEI and DVB/Carboxene/PDMS fibres. The response of the PEI fibre only reaches about $95 \%$ of the DVB/Carboxene/PDMS fibre response of tri- $\mathrm{ClB}, 150 \%$ of the response of tetra- $\mathrm{ClB}$, about $325 \%$ of the response of penta-ClB, and about $1100 \%$ of 
the response of hexa-ClB (see Figure 5). As for $\mathrm{HCH}$ isomers, the PEI fibre performed with noticeably higher responses than DVB/Carboxene/PDMS fibre. For some of the $\mathrm{HCH}$ isomers, the difference was several times higher (see Figure 5).

There is a validated procedure in our laboratory for $\mathrm{HCH}$ and $\mathrm{ClB}$ determination in water based on $100 \mu \mathrm{m}$ PDMS fibre. This procedure fulfils the required LOQ and LOD and employs a $50 \mathrm{~min}$ long extraction time. The PEI fibre reaches the corresponding response of a $100 \mu \mathrm{m}$ PDMS fibre $\left(1 \times 10^{7}\right.$ of arbitrary units) already within a 10 min extraction time.

These results are very promising in terms of the applicability of PEI fibres in the analysis of organochlorinated pesticides. However, long-term performance tests of PEI fibres are still missing. This could be a weak point in the involvement of nanoscaled sorbents in SPME, as the fibres can quickly lose the flexibility during GC temperature cycles. On the other hand, there was no visible damage to the PEI fibre after the 24 injections conducted in this study.

The other parameter, which remains unclear for the laboratory preparation of SPME PEI fibres for routine analytical purposes, is the fibre-to-fibre reproducibility of sorption properties. This aspect could be solved by using fibre quality control samples at the beginning of the work with a new fibre.

\section{Conclusions}

The feasibility of lab-scale preparation of several pieces of SPME PEI fibres by electrospinning has been proven. The prepared electrospun PEI nanofibres had better performance than three commercial SPME fibres in terms of the GCMS/MS system response for representatives of organochlorinated pesticides $(\mathrm{HCH}, \mathrm{ClB})$. Therefore, the extraction time could be shortened from 50 to $10 \mathrm{~min}$ by switching the PEI fibre for the $100 \mu \mathrm{m}$ PDMS fibre while maintaining the required sensitivity. This practically means considerable reduction of one-sample analysis time to the GC runtime (slightly over $20 \mathrm{~min}$ ). Fibre conditioning and another sample extraction can be done by GC-MS/MS autosampler in parallel during the GC runtime of the previous sample. As a result, 50 samples could be measured daily with PEI fibre instead of 20 samples with $100 \mu \mathrm{m}$ PDMS fibre.

Fast sorption and cheap, fast, and easy production of PEI nanofibres are the most beneficial properties in their application as sorbents in the SPME fibres. Based on obtained data, polyetherimide nanofibres as sorbent used in analytical chemistry deserve more detailed future research. Currently, only very few laboratories care about producing their own lab-made SPME fibres. However, with the global availability of all of the necessary components, the concept of lab-made SPME fibres is becoming a more valid option.

\section{Competing Interests}

The authors declare that they have no competing interests.

\section{Acknowledgments}

The research in this paper was supported by the project OPR\& DI, Institute for Nanomaterials, Advanced Technologies and
Innovation (CZ.1.05/2.1.00/01.0005), National Programme for Sustainability I (L01201). The work of Antoš Vojtěch was supported by the Ministry of Education of the Czech Republic within the SGS Project no. 21066/115 of the Technical University of Liberec. The authors acknowledge the assistance provided by the Research Infrastructure NanoEnviCz, supported by the Ministry of Education, Youth and Sports of the Czech Republic under Project no. LM2015073.

\section{References}

[1] G. Ouyang and J. Pawliszyn, "SPME in environmental analysis," Analytical and Bioanalytical Chemistry, vol. 386, no. 4, pp.10591073, 2006.

[2] S. Risticevic, Y. Chen, L. Kudlejova et al., "Protocol for the development of automated high-throughput SPME-GC methods for the analysis of volatile and semivolatile constituents in wine samples," Nature Protocols, vol. 5, no. 1, pp. 162-176, 2010.

[3] J. Pawliszyn, Handbook of Solid Phase Microextraction, Elsevier, 2011.

[4] P. Viñas, N. Campillo, N. Martínez-Castillo, and M. HernándezCórdoba, "Solid-phase microextraction on-fiber derivatization for the analysis of some polyphenols in wine and grapes using gas chromatography-mass spectrometry," Journal of Chromatography A, vol. 1216, no. 9, pp. 1279-1284, 2009.

[5] H.-G. Schmarr, W. Sang, S. Ganß et al., "Analysis of aldehydes via headspace SPME with on-fiber derivatization to their $O$-(2,3,4,5,6-pentafluorobenzyl)oxime derivatives and comprehensive 2D-GC-MS," Journal of Separation Science, vol. 31, no. 19, pp. 3458-3465, 2008.

[6] S. Risticevic, V. H. Niri, D. Vuckovic, and J. Pawliszyn, "Recent developments in solid-phase microextraction," Analytical and Bioanalytical Chemistry, vol. 393, no. 3, pp. 781-795, 2009.

[7] B. Bojko, E. Cudjoe, G. A. Gómez-Ríos et al., "SPME-Quo vadis?" Analytica Chimica Acta, vol. 750, pp. 132-151, 2012.

[8] H. Lord and J. Pawliszyn, "Evolution of solid-phase microextraction technology," Journal of Chromatography A, vol. 885, no. 1-2, pp. 153-193, 2000.

[9] M. T. Jafari, M. Saraji, and H. Sherafatmand, "Polypyrrole/montmorillonite nanocomposite as a new solid phase microextraction fiber combined with gas chromatographycorona discharge ion mobility spectrometry for the simultaneous determination of diazinon and fenthion organophosphorus pesticides," Analytica Chimica Acta, vol. 814, pp. 69-78, 2014.

[10] X. Hou, L. Wang, X. Tang, C. Xiong, Y. Guo, and X. Liu, "Application of a $\beta$-cyclodextrin/graphene oxide-modified fiber for solid-phase microextraction of six fragrance allergens in personal products," The Analyst, vol. 140, no. 19, pp. 6727-6735, 2015.

[11] J. López-Darias, V. Pino, Y. Meng, J. L. Anderson, and A. M. Afonso, "Utilization of a benzyl functionalized polymeric ionic liquid for the sensitive determination of polycyclic aromatic hydrocarbons; parabens and alkylphenols in waters using solidphase microextraction coupled to gas chromatography-flame ionization detection," Journal of Chromatography A, vol. 1217, no. 46, pp. 7189-7197, 2010.

[12] X. Wang, B. Liu, Q. Lu, and Q. Qu, "Graphene-based materials: fabrication and application for adsorption in analytical chemistry," Journal of Chromatography A, vol. 1362, pp. 1-15, 2014.

[13] A. Kumar, Gaurav, A. K. Malik, D. K. Tewary, and B. Singh, "A review on development of solid phase microextraction fibers by 
sol-gel methods and their applications," Analytica Chimica Acta, vol. 610, no. 1, pp. 1-14, 2008.

[14] H. Bagheri and A. Aghakhani, "Polyaniline-nylon-6 electrospun nanofibers for headspace adsorptive microextraction," Analytica Chimica Acta, vol. 713, pp. 63-69, 2012.

[15] H. Bagheri, H. Najarzadekan, and A. Roostaie, "Electrospun polyamide-polyethylene glycol nanofibers for headspace solidphase microextration," Journal of Separation Science, vol. 37, no. 14, pp. 1880-1886, 2014.

[16] H. Bagheri, A. Akbarinejad, and A. Aghakhani, "A highly thermal-resistant electrospun-based polyetherimide nanofibers coating for solid-phase microextraction," Analytical and Bioanalytical Chemistry, vol. 406, no. 8, pp. 2141-2149, 2014.

[17] J. Niu, Z. Li, H. Yang et al., "A water resistant solid-phase microextraction fiber with high selectivity prepared by a metal organic framework with perfluorinated pores," Journal of Chromatography A, vol. 1441, pp. 16-23, 2016.

[18] N. P. Brunton, D. A. Cronin, and F. J. Monahan, "The effects of temperature and pressure on the performance of Carboxen/PDMS fibres during solid phase microextraction (SPME) of headspace volatiles from cooked and raw turkey breast," Flavour and Fragrance Journal, vol. 16, no. 4, pp. 294302, 2001.

[19] S. L. Chong, D. Wang, J. D. Hayes, B. W. Wilhite, and A. Malik, "Sol-Gel coating technology for the preparation of solid-phase microextraction fibers of enhanced thermal stability," Analytical Chemistry, vol. 69, no. 19, pp. 3889-3898, 1997.

[20] S. Wacławek, V. Antoš, P. Hrabák, M. Černík, and D. Elliott, "Remediation of hexachlorocyclohexanes by electrochemically activated persulfates," Environmental Science and Pollution Research, vol. 23, no. 1, pp. 765-773, 2016.

[21] A. A. Boyd-Boland, S. Magdic, and J. B. Pawliszyn, "Simultaneous determination of 60 pesticides in water using solid-phase microextraction and gas chromatography-mass spectrometry," Analyst, vol. 121, no. 7, pp. 929-938, 1996.

[22] R. Boussahel, S. Bouland, K. M. Moussaoui, M. Baudu, and A. Montiel, "Determination of chlorinated pesticides in water by SPME/GC," Water Research, vol. 36, no. 7, pp. 1909-1911, 2002.

[23] C. Dong, Z. Zeng, and M. Yang, "Determination of organochlorine pesticides and their derivations in water after HSSPME using polymethylphenylvinylsiloxane-coated fiber by GC-ECD," Water Research, vol. 39, no. 17, pp. 4204-4210, 2005.

[24] A. Menezes Filho, F. N. dos Santos, and P. A. de Paula Pereira, "Development, validation and application of a methodology based on solid-phase micro extraction followed by gas chromatography coupled to mass spectrometry (SPME/GC-MS) for the determination of pesticide residues in mangoes," Talanta, vol. 81, no. 1-2, pp. 346-354, 2010.

[25] Y. Picó, M. Fernández, M. J. Ruiz, and G. Font, "Current trends in solid-phase-based extraction techniques for the determination of pesticides in food and environment," Journal of Biochemical and Biophysical Methods, vol. 70, no. 2, pp. 117-131, 2007. 

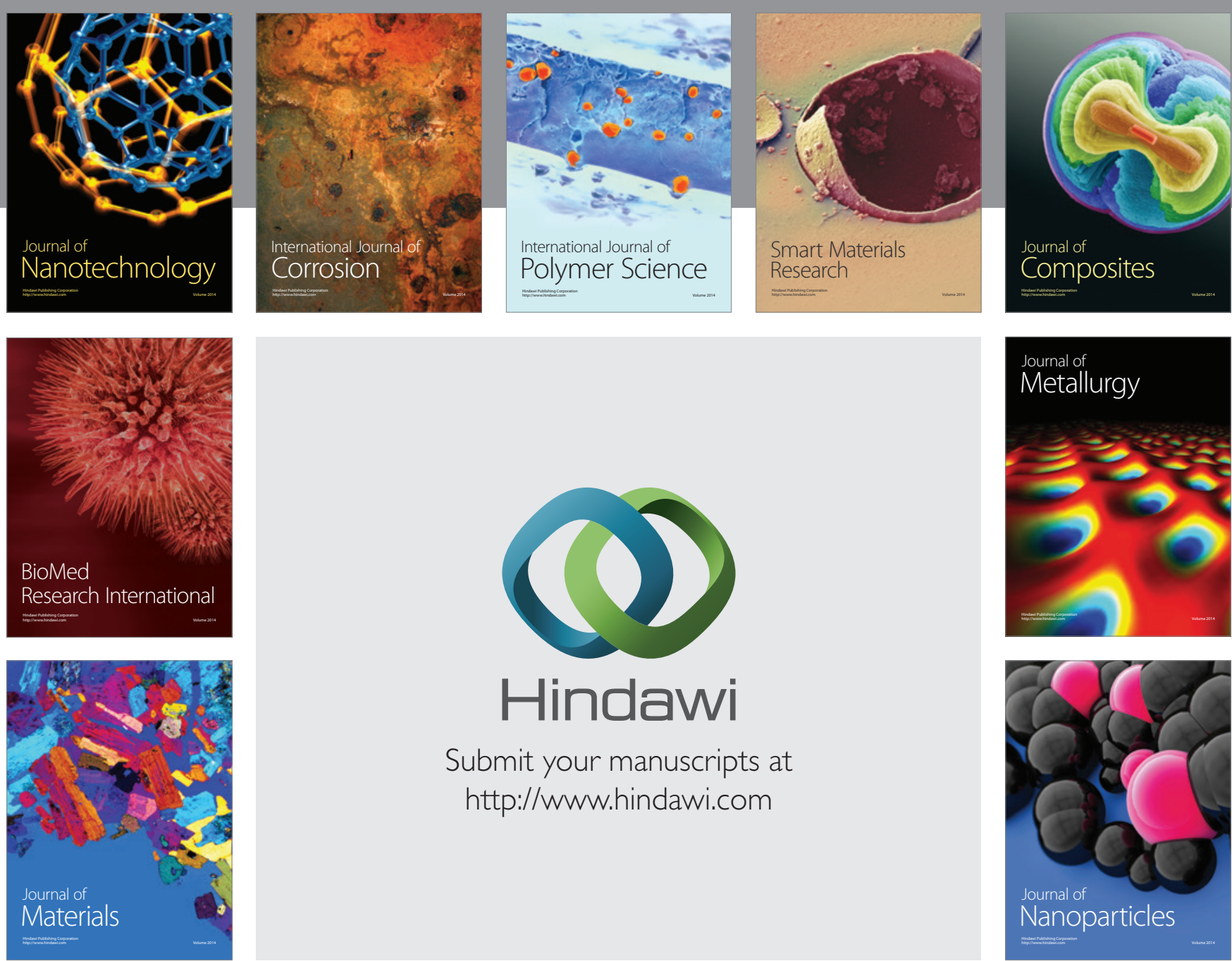

\section{Hindawi}

Submit your manuscripts at

http://www.hindawi.com

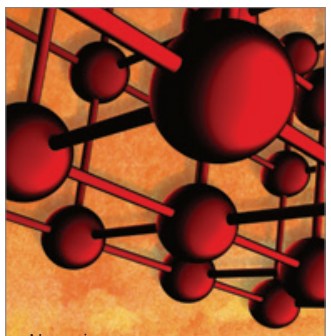

Materials Science and Engineering
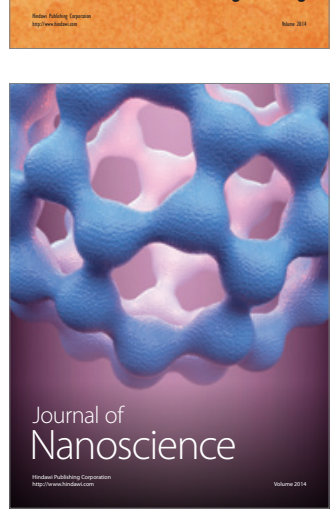
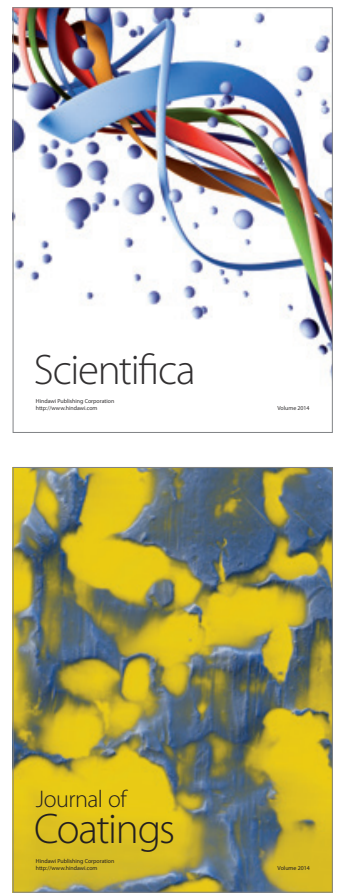
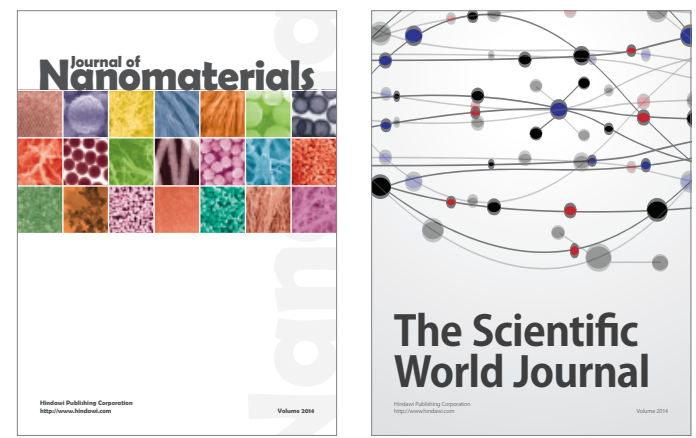

The Scientific World Journal
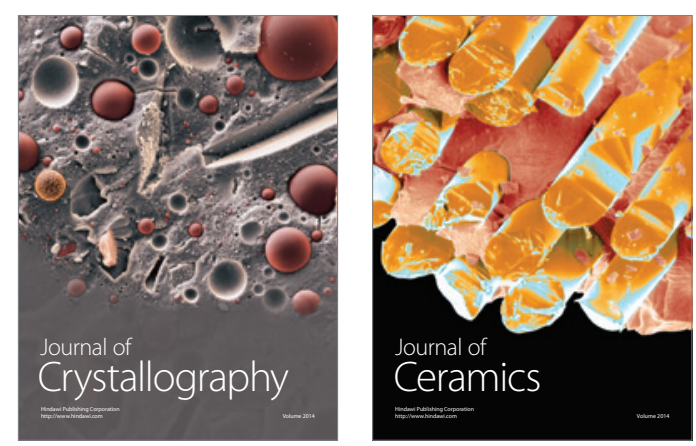
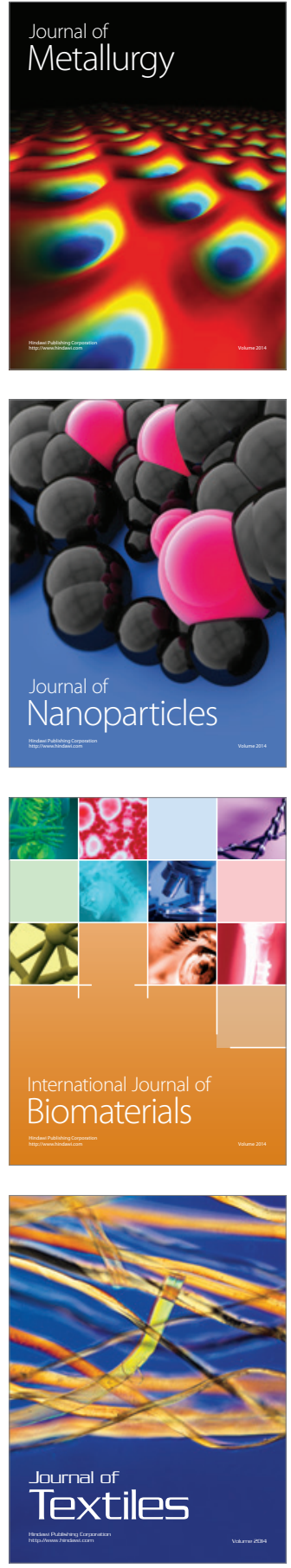